\title{
Functional Prognosis after Treatment of Spinal Dural Arteriovenous Fistulas
}

\author{
Satoshi USHIKOSHI, Kazutoshi HIDA*, Yoichi KIKUCHI, Kazuo MIYASAKA, \\ Toshinobu IWASAKI*, and Hiroshi ABE*
}

Departments of Radiology and *Neurosurgery, Hokkaido University School of Medicine, Sapporo

\begin{abstract}
Functional prognosis after treatment for spinal dural arteriovenous fistulas (SDAVFs) was retrospectively analyzed in 13 consecutive patients aged 38 to 73 years (mean 57 years) treated during the last 5 years. The duration of symptoms before diagnosis ranged from 3 to 72 months (mean 23 months). Neurological symptoms were examined before and 6 months after the treatment. Seven patients underwent embolization as the initial treatment. In four of six patients, N-butyl 2-cyanoacrylate (NBCA) embolization achieved complete obliteration of SDAVF. The other two patients with incomplete embolization and one embolized with polyvinyl alcohol particles underwent subsequent surgical treatment. Six patients were treated by direct surgery. Complete disappearance of SDAVF was confirmed in all nine patients treated surgically. Improvement of gait and micturition disturbance after the treatment was noted in six of 10 and three of six patients, respectively. Long duration of symptoms and high grade of neurological symptoms were associated with a poor functional outcome. NBCA embolization and surgery are curative treatments for SDAVF, but the functional prognosis is not always satisfactory. Embolization is the first choice of treatment for SDAVF because it is less invasive and relatively safe. However, when complete obliteration is not achieved, prompt surgery is recommended because a long duration of symptoms will result in a poor functional prognosis.
\end{abstract}

Key words: spinal cord, dural arteriovenous fistula, prognosis

\section{Introduction}

Spinal dural arteriovenous fistula (SDAVF) is one of the most common types of spinal vascular abnormalities. ${ }^{11,16,17)}$ SDAVF is a direct AVF within the dura fed by one or more radiculomeningeal arterioles and ending in a single draining vein, without an intervening capillary plexus, as shown by microangiographic and some pathological studies. ${ }^{3,14,18)}$ Most SDAVFs are located in the thoracolumbar areas. The retrograde venous drainage flows via a radicular vein into the dilated perimedullary venous system. Usually, outflow to the extradural venous plexus via the radicular veins of neighboring segments cannot be observed over a long distance for unclear reasons, and the draining vein courses upward to the cervical or the craniocervical region.

Chronic venous hypertension is the most important pathophysiological factor contributing to neurological deficits in patients with SDAVF. ${ }^{5,9,10,17)}$

Received August 13, 1998; Accepted October 26, 1998
Reflux of the shunt flow into the perimedullary venous system and outflow obstruction increase the spinal venous pressure and decrease the arteriovenous pressure gradients, which can lead to chronic hypoxia with progressive and subsequently irreversible damage to the medullary matter. Anatomical cure of SDAVF can be obtained by either embolization or surgery, but the functional outcome is not always satisfactory. ${ }^{1,12,15,18)}$

We retrospectively reviewed our series of cases of SDAVF and analyzed the factors correlated with the functional prognosis.

\section{Materials and Methods}

Thirteen consecutive patients with SDAVF, nine males and four females, aged from 38 to 73 years (mean 57 years) were treated during the last 5 years (Table 1). The duration of symptoms before diagnosis ranged from 3 to 73 months (mean 23 months). The SDAVF was located in the cervical region in two patients, thoracic region in seven patients, and lumbar region in four patients. All patients with 
Table 1 Summary of patients

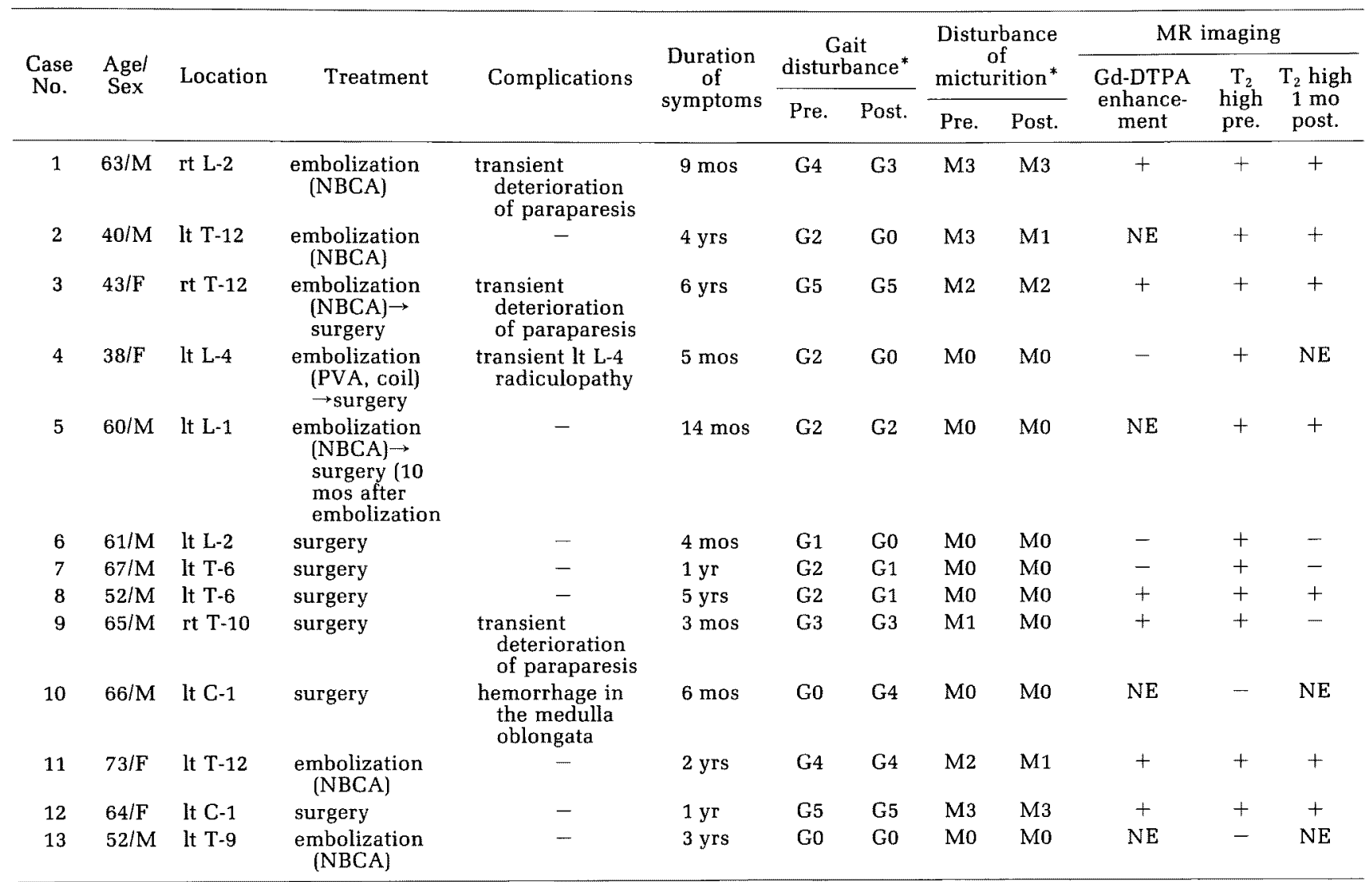

${ }^{*}$ According to the scale of Aminoff and Logue. ${ }^{21}$ Gd-DTPA: gadolinium-diethylenetriaminepenta-acetic acid, MR: magnetic resonance, NBCA: n-butyl 2-cyanoacrylate, NE: not examined, post.: after treatment, pre.: before treatment, PVA: polyvinyl alcohol particles.

neurological deficits were indicated for the treatment. Patients without neurological symptoms were also treated when the venous drainage flowed into the medullary vein. The choice of treatment for each patient was decided by consultation among interventional neuroradiologists and neurosurgeons.

Embolization was performed with variable stiffness microcatheters and guidewires under local anesthesia and systemic heparinization in all patients. The feeding artery was selectively catheterized and embolized after confirming that no spinal cord artery arose from the same pedicle as the feeding artery. As embolic materials, n-butyl 2cyanoacrylate (NBCA) was used when complete and permanent obliteration was attempted. Polyvinyl alcohol (PVA) particles or metallic coils were used when embolization was performed as a preoperative treatment to reduce the shunt flow. Heparinization was not performed after the embolization.

The surgical procedure was as follows. A laminectomy to extend about one level above and below the level of the SDAVF was performed and a midline dural incision was made. The arterialized medullary draining vein was exposed and interrupted as it entered the subarachnoid space. Excision of the AVF was not performed.

Functional outcome was estimated 6 months after the treatment according to the scale of Aminoff and Logue. ${ }^{2)}$ Patient age, duration of symptoms before treatment, functional status before treatment, and magnetic resonance (MR) imaging findings (intramedullary high signal on $\mathrm{T}_{2}$-weighted images and gadolinium-diethylenetriaminepenta-acetic acid [Gd-DTPA] enhancement) were correlated with the functional prognosis.

\section{Results}

\section{Anatomical results}

Seven patients underwent embolization as the initial treatment. Embolization used NBCA in six patients, and complete occlusion of the SDAVF was achieved in four. Two other patients with unsuccessful embolization underwent surgery. Preopera- 
tive embolization using particles and coils was performed in one patient with high flow SDAVF to reduce the shunt flow. Six patients underwent direct surgery. Complete occlusion of SDAVF was obtained in all nine surgically treated patients.

\section{Complications}

Transient neurological deterioration occurred in one patient 1 day after successful embolization with NBCA. Thrombosis of the draining vein was suspected to be the cause because of the delayed onset.

Transient neurological deterioration due to surgical manipulation occurred in three patients. One patient who presented with radiculopathy after surgery achieved complete resolution of the symptoms. The other two patients who experienced aggravation of myelopathy after the surgery showed gradual improvement. However, their final functional status was in the same grade as the preoperative status.

Permanent neurological complications occurred in one patient. This 66-year-old male with SDAVF fed by left C-1 segmental artery suffered hemorrhage in the medulla oblongata 1 day after the surgery due to an unknown cause and difficulty in swallowing and left hemiparesis persisted.

\section{Clinical outcome}

One patient who underwent surgery for recurrence 10 months after embolization was excluded from this assessment.

Gait disturbance was present in 10 patients. After treatment, six patients had improvement (all five grade 1-2 patients and one of the five grade 3-5

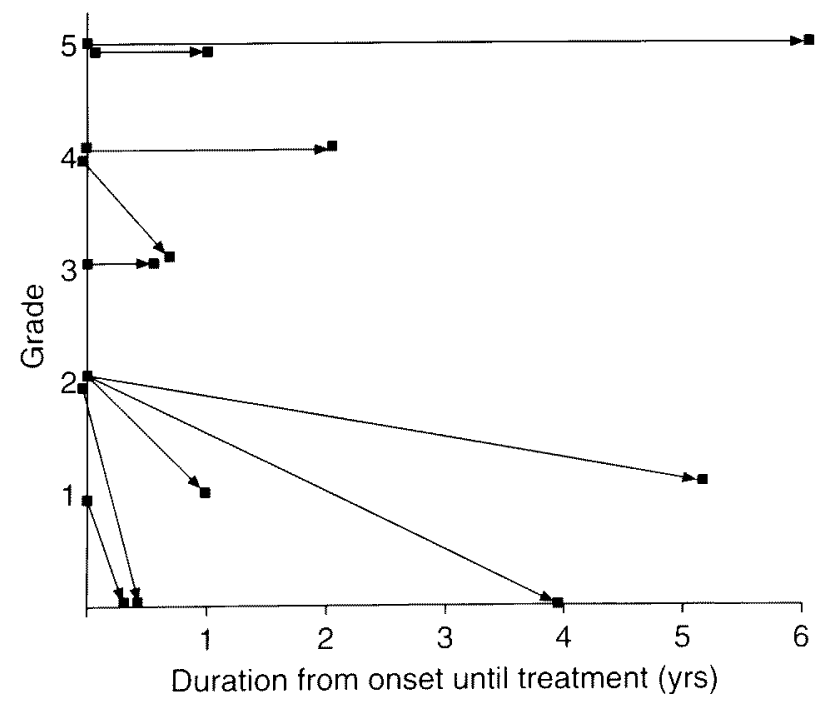

Fig. 1 Changes in gait disturbance before and after treatment.

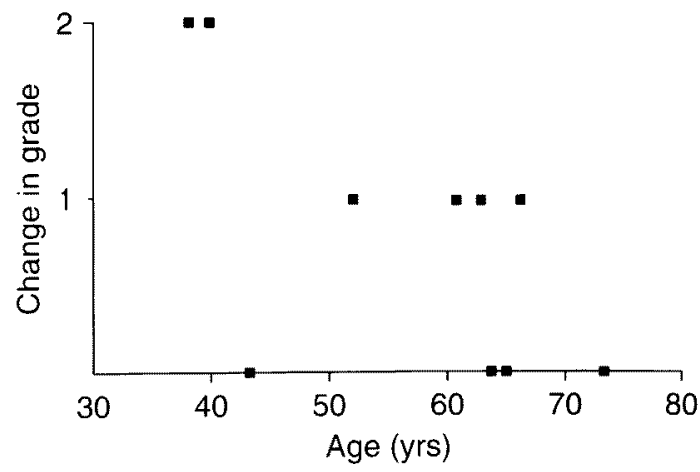

Fig. 2 Changes in grade of gait disturbance and age of patients.

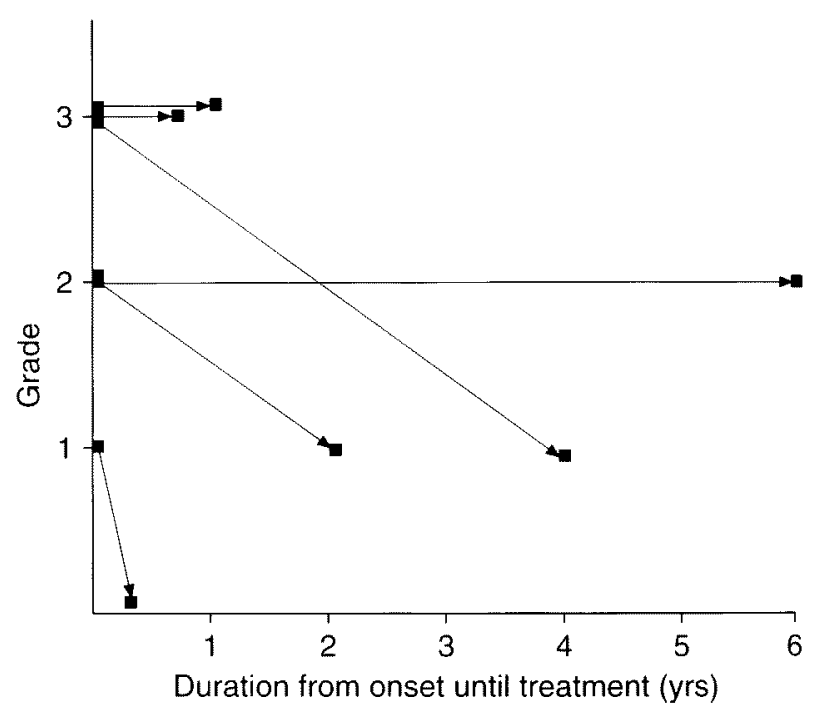

Fig. 3 Changes in disturbance of micturition before and after treatment.

patients), and the other four patients remained in the same grade. A shorter duration of symptoms tended to indicate a more favorable recovery (Fig. 1). Older patients also tended to have a poorer recovery (Fig. 2).

$\mathrm{T}_{2}$-weighted MR imaging before treatment showed an intramedullary high signal in all 10 patients. MR imaging with Gd-DTPA demonstrated abnormal enhancement in the spinal cord in six of nine patients. Only two of these patients showed improvement after treatment, but all three patients without GdDTPA enhancement showed improvement (Table 1).

Disturbance of micturition was noted in six patients. Three patients showed improvement after treatment. Two of the three grade 1-2 patients experienced improvement after treatment but only one patient (aged 40 years) of the three grade 3 patients 

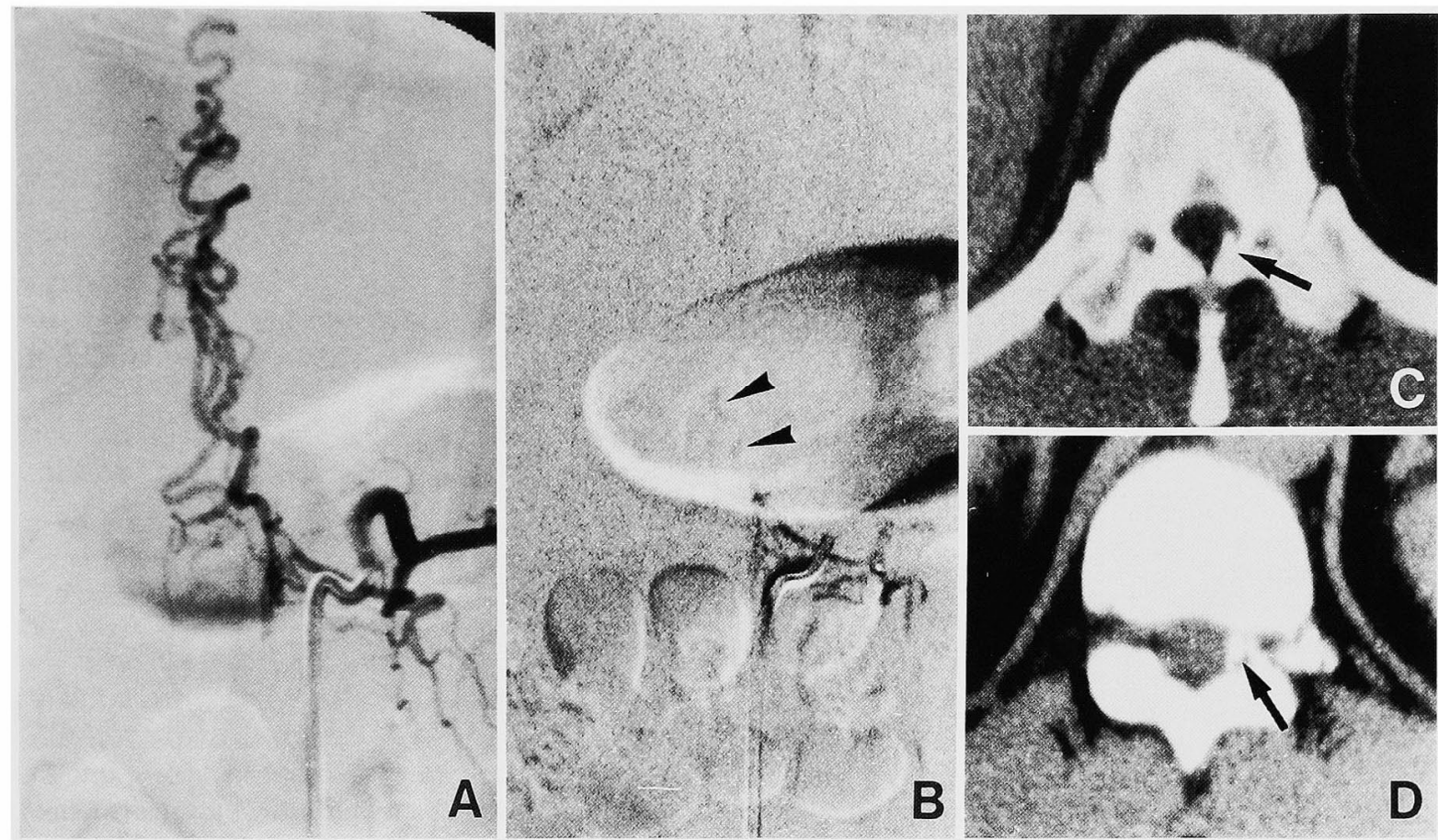

Fig. 4 Case 2. Angiograms of the left T-11 intercostal artery, showing the spinal dural arteriovenous fistula (A), and after superselective injection of n-butyl 2-cyanoacrylate (NBCA) showing the cast of NBCA in the proximal portion of the draining vein (B: arrowheads). Immediate postembolization computed tomography scans at the T-10 level (C) and T10-11 intervertebral level (D), showing the NBCA inside the dura (arrow).

showed improvement (Fig. 3).

\section{Illustrative Cases}

Case 2: A 40-year-old male presented with a 4-year history of progressive gait disturbance, sensory disturbance of the lower extremities, and urinary retention. Spinal angiography demonstrated a SDAVF supplied by the left T-11 intercostal artery (Fig. 4A), which was selectively catheterized and embolized with NBCA (Fig. 4B). Immediate postembolization computed tomography (CT) clearly showed the NBCA cast inside the dura (Fig. 4C, D). The symptoms improved gradually and only mild urinary retention was noted 6 months after embolization. Follow-up angiography performed 1 year and 6 months after the embolization showed no evidence of recurrence.

Case 5: A 60-year-old male presented with a 14month history of gait disturbance, sensory disturbance of the lower extremities, and urinary frequency. Spinal angiography demonstrated a SDAVF supplied by the left L-1 lumbar artery (Fig. 5A), which was selectively catheterized and embolized with NBCA. Immediate postembolization an- giography showed disappearance of the SDAVF (Fig. 5B). The symptoms improved after embolization, but again deteriorated 6 months after embolization. Follow-up angiography 8 months after embolization revealed the recurrent SDAVF due to the development of collaterals (Fig. 5C, D). Although the patient was successfully treated by surgery, his symptoms did not improve in the 2-year follow-up period.

\section{Discussion}

Surgical treatment of SDAVF is relatively easy and safe compared with other vascular malformations of the spinal cord. The most common surgical procedure is simple interruption of the communicating vessels between the AVF and coronal venous plexus. ${ }^{118)}$ In our series, surgery achieved complete disappearance of the SDAVF in all nine patients. Surgery is a curative treatment when there is only the intradural venous drainage. However, when both intra- and extradural venous drainage is present preoperatively, the shunt flow can persist not only into the extradural venous system but also into the intradural medullary vein. ${ }^{11}$ More complicated and 


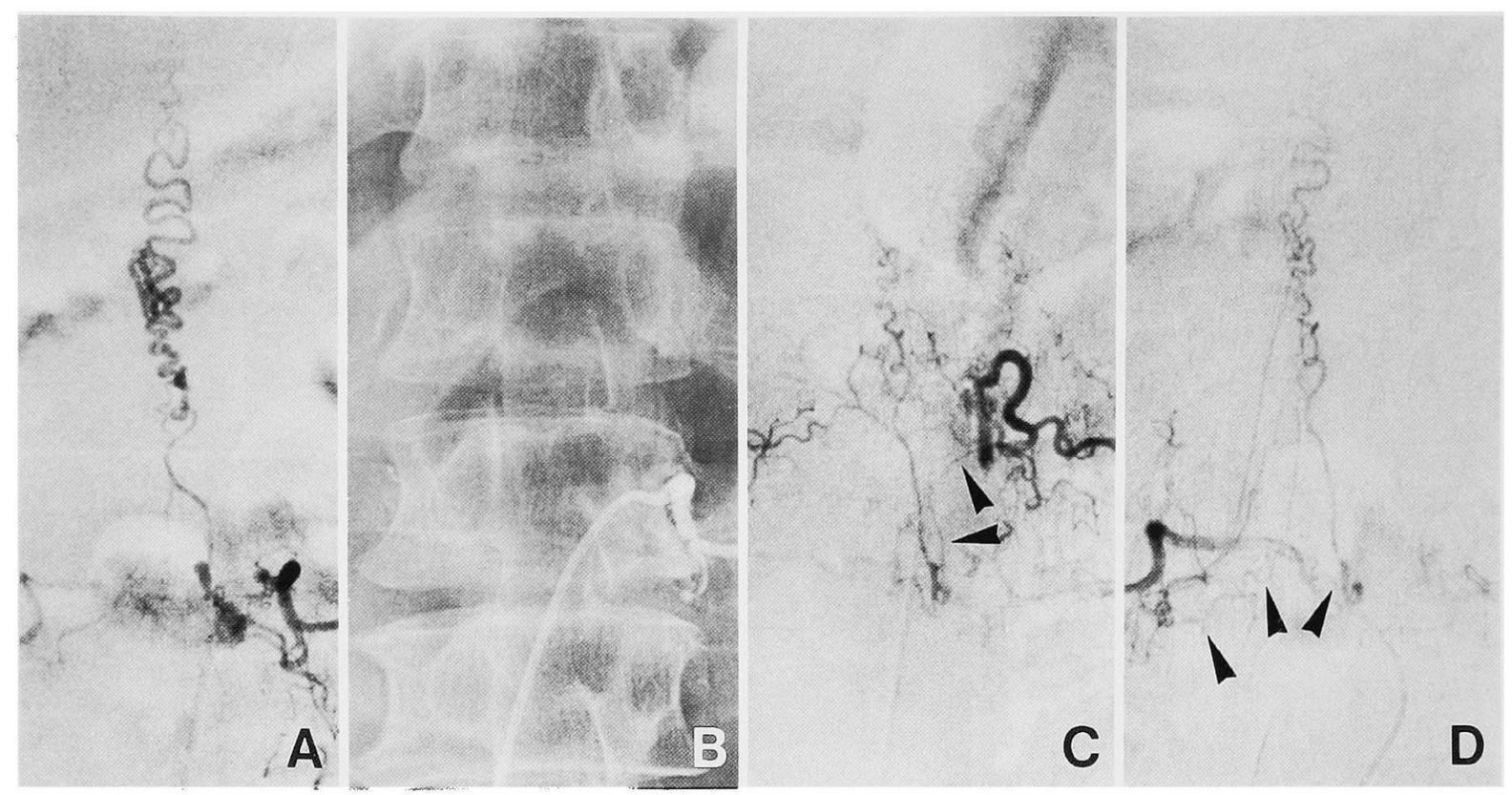

Fig. 5 Case 5. Angiograms of the left L-1 lumbar artery, showing the spinal dural arteriovenous fistula (SDAVF) (A), and postembolization showing no opacification of the SDAVF (B). Angiograms of the left T-12 intercostal artery (C) and right L-1 lumbar artery (D) performed 8 months after embolization, showing the recurrent SDAVF supplied by the collateral vessels (arrowheads).

invasive surgical procedures, such as complete excision of the fistula or interruption of all the intraand extradural venous drainage, are recommended in patients with extradural venous drainage.

Since the introduction of variable stiffness microcatheters and guidewires, embolization has become one of the important choices for the treatment of SDAVF. The incidence of recanalization is high when particulate embolic materials such as PVA particles are used ${ }^{8)}$ However, due to the recent development of techniques and NBCA as a permanent liquid embolic material, which can sufficiently penetrate the lesion, the rate of complete and permanent occlusion has improved significantly. Thirty-three of 38 cases of SDAVF treated by acrylic materials and variable stiffness microcatheters were successfully occluded with no technical complications. ${ }^{15)}$ Although the success rate of embolization is still lower than that of surgical treatment, embolization is less invasive and can be performed relatively easily with a low complication rate. Extradural venous drainage, which requires an additional complicated surgical procedure, can be occluded by the same embolization procedure. Subsequent deterioration of symptoms can occur after treatment due to progressive venous thrombosis, but systemic heparinization can be started immedi- ately after the embolization in such a situation.

SDAVF are usually low flow type AVF, and incomplete embolization is mainly due to proximal occlusion of the feeding artery. ${ }^{15)}$ In our two patients with unsuccessful embolization, injected NBCA was believed to have stopped proximal to the fistula and not penetrated the fistula. To achieve the complete obliteration of SDAVF, the liquid embolic material should penetrate distally to the proximal portion of the draining vein. If the embolic material does not penetrate the fistulous portion sufficiently, recurrence may occur due to collateralization. However, assessment of distal penetration of the material may be difficult at the time of embolization. Five of 33 patients experienced recurrence during the followup period after initial "adequate" embolization because of collateralization. ${ }^{15)} \mathrm{MR}$ imaging is not useful to evaluate the complete obliteration of AVF. Absence of flow void does not exclude the presence of residual AVF. ${ }^{7)}$ Moreover, only three of our 11 patients with an intramedullary high signal on $\mathrm{T}_{2}$ weighted MR images showed the disappearance of the high signal within 1 month after successful treatment (Table 1). Immediate postembolization CT is useful to confirm the location of the NBCA cast and complete cure, as in our Case 2. ${ }^{4}$

Embolization with NBCA and surgery are both 
curative treatments for SDAVF. However, anatomical cure does not always lead to a functional recovery. Follow up of 46 patients with SDAVF treated surgically found improvement of disturbed gait in 20 of 31 grade 3-5 (according to the scale of Aminoff and Logue ${ }^{2)}$ ) patients, and 12 of 15 grade 1-2 patients. ${ }^{181}$ Improvement by two grades was seen in only seven patients and by one grade in 25 patients. Follow up of 19 patients found neurological improvement in eight patients but the same neurological status in 11 patients after successful surgical treatment. ${ }^{1)}$ Statistical analysis of 39 cases showed that a patient under 70 years old, who is treated within 2 years and 6 months of onset, with slight or moderate gait or urinary disturbance, and without absence of deep tendon reflexes in the lower extremities, is expected to have a good functional prognosis after treatment. ${ }^{12)}$ In our series, six of 10 patients with gait disturbance and three of six patients with disturbance of micturition showed improvement after treatment. Younger patients with a shorter duration of symptoms and with a lower grade before treatment tended to have better functional recovery. The most important point for obtaining favorable functional outcome is diagnosis of this disease at an early stage of myelopathy and appropriate treatment before the spinal cord suffers irreversible damage caused by venous hypertension.

$\mathrm{T}_{2}$-weighted MR imaging showed an intramedullary high signal in all patients with neurological symptoms in our series, but it was difficult to determine whether the increased $\mathrm{T}_{2}$ signal indicated reversible edema caused by venous hypertension or irreversible venous infarction. ${ }^{6,7,13)}$ However, patients with abnormal enhancement in the spinal cord on MR imaging with Gd-DTPA had poorer functional improvement after treatment. Although the number of patients is too small to reach a definite conclusion, MR imaging with Gd-DTPA may be useful for the diagnosis of SDAVF and the prediction of functional outcome.

Accordingly, we think that embolization is the first choice of treatment for SDAVF when there is no spinal cord artery arising from the same pedicle as the feeder to the lesion. If complete obliteration is not achieved by embolization, prompt surgery is recommended because a long duration of symptoms will result in a poor functional prognosis. CT may be useful in confirming complete cure immediately after embolization with NBCA.

\section{References}

1) Afshar JKB, Doppman JL, Oldfield EH: Surgical interruption of intradural draining vein as curative treatment of spinal dural arteriovenous fistulas. J Neurosurg 82: 196-200, 1995

2) Aminoff MJ, Logue V: The prognosis of patients with spinal vascular malformations. Brain 97: 211-218, 1974

3) Benhaiem N, Poirier J, Hurth M: Arteriovenous fistulae of the meninges draining into the spinal veins: a histological study of 28 cases. Acta Neuropathol (Berl) 62: 103-111, 1983

4) Cognard C, Miaux Y, Pierot L, Weill A, Martin N, Chiras J: The role of CT in evaluation of the effectiveness of embolisation of spinal dural arteriovenous fistulae with $\mathrm{N}$-butyl cyanoacrylate. Neuroradiology 38: 603-608, 1996

5) Criscuolo GR, Oldfield EH, Doppman JL: Reversible acute and subacute myelopathy in patients with dural arteriovenous fistulas: Foix-Alajouanine syndrome reconsidered. J Neurosurg 70: 354-359, 1989

6) Dormont D, Gelbert F, Assouline E, Reizine D, Helias A, Riche MC, Chiras J, Bories J, Merland JJ: MR imaging of spinal cord arteriovenous malformations at 0.5 T: study of 34 cases. AJNR Am J Neuroradiol 9: 833-838, 1988

7) Gilbertson JR, Miller GM, Goldman MS, Marsh WR: Spinal dural arteriovenous fistulas: MR and myelographic findings. AJNR Am J Neuroradiol 16: 20492057, 1995

8) Hall WA, Oldfield EH, Doppman JL: Recanalization of spinal arteriovenous malformations following embolization. J Neurosurg 70: 714-720, 1989

9) Hassler W, Thron A, Grote EH: Hemodynamics of spinal dural arteriovenous fistulas: an intraoperative study. J Neurosurg 70: 360-370, 1989

10) Hurst RW, Kenyon LC, Lavi E, Raps EC, Marcotte P: Spinal dural arteriovenous fistula: the pathology of venous hypertensive myelopathy. Neurology 45: 1309-1313, 1995

11) Kendall BE, Logue V: Spinal epidural angiomatous malformations draining into intrathecal veins. Neuroradiology 13: 181-189, 1977

12) Kohno $M$, Takahashi $H$, Kitanaka $C$, Sasaki $T$, Ishijima B: Functional prognosis after treatment of spinal radiculomeningeal arteriovenous malformations. Surg Neurol 43: 453-458, 1995

13) Larsson E-M, Desai P, Hardin CW, Story J, Jinkins JR: Venous infarction of the spinal cord resulting from dural arteriovenous fistula: MR imaging findings. AJNR Am J Neuroradiol 12: 739-743, 1991

14) McCutcheon IE, Doppman JL, Oldfield EH: Microvascular anatomy of dural arteriovenous abnormalities: a microangiographic study. J Neurosurg 84: 215-220, 1996

15) Niimi $Y$, Berenstein A, Setton A, Neophytides A: Embolization of spinal dural arteriovenous fistulae: result and follow-up. Neurosurgery 40: 675-683, 1997

16) Riche MC, Reizine D, Melki JP, Merland JJ: Classification of spinal cord vascular malformations. Radiat Med 3: 17-24, 1985

17) Rosenblum B, Oldfield EH, Doppman JL, Chiro GD: Spinal arteriovenous malformations: a comparison 
of dural arteriovenous fistulas and intradural AVM's in 81 patients. J Neurosurg 67: 795-802, 1997

18) Symon L, Kuyama H, Kendall B: Dural arteriovenous malformations of the spine: clinical features and surgical results in 55 cases. J Neurosurg 60: 238-247, 1984

Address reprint requests to: S. Ushikoshi, M.D., Department of Radiology, University of Hokkaido School of Medicine, North-15, West-7, Kita-ku, Sapporo 0608638, Japan.

\section{Commentary}

Arteriovenous malformations (AVMs) occur in the central nervous system and are fairly common in the brain. To have them occur in the spinal cord is not nearly as common and can be confused with certain angiomas within the cord itself. Having a surface dural $A V M$ and/or arteriovenous fistula $(A V F)$ is rare. For the patients to have symptoms for quite sometime before being diagnosed is not uncommon. Fortunately, some of the newer magnetic resonance imaging (MRI) studies are finding these lesions earlier. But they have been disappointing on occasion with persons who have suffered a progressive paraparetic syndrome and who have had MRI screening studies which have failed to pick up the lesion. Only after having followed through with old fashioned myelography and special CAT scanning have the lesions been identified. Then angiography will confirm the presence of the "AVM" or "AVF." We are in agreement that chronic venous hypertension produced by these lesions is the most important pathophysiological factor contributing to the neurologic deficit.

We agree that the first treatment trial should be with intravascular embolization. Admittedly, only about $60-70 \%$ of the patients have a vessel which can be embolized to correct the problem. In nearly a third of the cases, there is no vessel that the angiographers can safely embolize. It is at that time they are treated surgically. When treated surgically, it is not that difficult a procedure. Most of these are in the thoracic area, most can have an adequate exposure to see the few abnormality, and obliterating the fistula with stripping away of some of the dilated venous abnormality is fairly straight forward under the microscope. The treatment clearly stops the progression of the disease. But as these authors found out, only half of the patients get substantial improvement in their neurologic deficit. We have these patients enter an aggressive rehabilitation program and followed by our experts in physiatry.

These authors do have some new important find- ings. They again point out the most important favorable functional outcome factor is diagnosis and treatment in the early stages of the myelopathy. They also have better recent MRIs to show an intramedullary high signal in all of the patients preoperatively with neurologic symptoms. When they did gadolinium enhancement and the intramedullary enhancement increased, those patients had a poor functional outcome. That is logical to us, though we do not have confirming data.

The authors are to be complimented on providing us with the most recent and complete review on this subject.

Thomas B. DUCKER, M.D., F.A.C.S. Department of Neurological Surgery Johns Hopkins University and University of Maryland Maryland, U.S.A.

This article discusses the functional prognosis of 13 patients who were successfully treated for spinal dural arteriovenous fistulas (SPDAVFs). The mean duration of symptoms prior to treatment was 23 months. Seven patients were treated initially with intraarterial embolization, three of whom required surgery for complete obliteration of the fistula. The remaining 6 patients underwent surgery as the primary treatment. Functional outcome was evaluated at 6 months. The study shows that obliteration of the fistula arrested or improved symptoms of gait and micturition disturbance in all patients. The degree of functional recovery was related to younger patient age, shorter duration of symptoms, and lesser degree of initial neurologic impairment.

Our experience with SPDAVFs is similar to that reported in this article. Unfortunately, the majority of our patients also present with a long duration of symptoms ranging from months to years and often, by the time the correct diagnosis is made, the patients have significant neurologic deficits. We treat SPDAVFs with either surgery or embolization, depending on the individual case after combined neurointerventional and neurosurgical consultation. We have also documented that the expected functional recovery is related to the duration of symptoms and the degree of neurologic deficit.

This article emphasizes the importance of a prompt diagnosis of SPDAVF. Often these lesions are misdiagnosed as inflammation, tumor, or demyelination of the spinal cord. A progressive myelopathy in a middle aged to elderly person (especially male) should prompt an evaluation for a SPDAVF. The initial diagnostic study should be a high-quality MRI. The spectrum of MRI findings is variable, consisting of increased cord signal on the $\mathrm{T}_{2}$-weighted images, cord 
swelling, abnormal flow voids, intramedullary enhancement, and abnormal venous enhancement. A combination of increased $T_{2}$ cord signal and either enlarged flow voids or abnormal enhancement of the coronal venous plexus is very suspicious for a SPDAVF and requires angiography for further evaluation. An increased $\mathrm{T}_{2}$ cord signal alone is nonspecific, but in the proper clinical setting should prompt a supine myelogram to evaluate for an enlarged coronal venous plexus (ref. 7 of this article). In summary, this study demonstrates that the most important aspect in the management of a patient with SPDAVF is a prompt diagnosis to allow treatment prior to permanent neurologic deterioration.

Robert J. ERNST, M.D.

Department of Radiology

John M. TEW, Jr., M.D.

Department of Neurosurgery

University of Cincinnati College of Medicine

Ohio, U.S.A.

The authors treated 13 patients with spinal dural arteriovenous fistulas (SDAVF). They found that younger patients with a shorter duration of myelopathy have better functional recovery. We agree with them that intramedullary enhancement on MRI, suggesting disrupted blood brain barrier, is a sign of poorer functional recovery. Although they concluded that endovascular intervention is the first choice for SDAVF, their successful obliteration rate is not high (4/6) and one patient suffered transient deterioration due to delayed venous thrombosis. As they stated, emboli should penetrate to the proximal portion of the draining system to obliterate the lesions completely, possibly aggravating spinal venous congestion. We think that surgical interruption is the most suitable therapeutic option for SDAVF and epidural coagulation of the lesions is essential. Without epidural procedures, some lesions may persist as SDAVF draining solely into the internal vertebral venous plexus, without perimedullary drainage.

Nobuo HASHIMOTO, M.D.

and Susumu MiYamoto, M.D.

Department of Neurosurgery

Kyoto University Medical School

Kyoto, Japan

The authors report $a$ series of 13 patients with spinal dural arteriovenous fistula (SDAVF), aged 38 to 73 years (mean 57 years) treated by endovascular surgery and/or microsurgery, and analyzed the factors correlated with the functional prognosis. The duration of symptoms before diagnosis ranged from 3 to 73 months (mean 23 months). The SDAVF was located in the cervical region in 2 patients, thoracic region in 7 patients, and lumbar region in 4 patients. The feeding artery was selectively catheterized and embolized after confirming that no spinal cord artery arose from the same pedicle as the feeding artery with NBCA. Surgical procedure was interruption of arterized medullary draining vein after laminectomy and dural incision. Functional outcome was estimated 6 months after treatment. The authors concluded that embolization is the first choice of treatments for SDAVF because it is less invasive and relatively safe, and that, when complete obliteration is not achieved, prompt surgery is recommended because a long duration of symptoms will result in a poor functional prognosis. It is becoming clearer that certain lesions are best treated by microsurgery and other lesions are best treated by endovascular techniques. However, there is an increasing number of lesions for which the best treatment is not obvious. Regardless of the strategies applied, it is critical that long-term follow-up monitoring for many years be used for these patients.

Tadashi KojIMA, M.D. Department of Neurosurgery Mie University School of Medicine Mie, Japan 